\title{
The clinical promise of immunotherapy in triple- negative breast cancer
}

This article was published in the following Dove Press journal: Cancer Management and Research

\author{
Praveen Vikas ${ }^{1,2}$ \\ Nicholas Borcherding ${ }^{2-5}$ \\ Weizhou Zhang ${ }^{2-5}$ \\ 'Department of Internal Medicine, \\ College of Medicine, University of \\ lowa, lowa City, IA, USA; ${ }^{2}$ Holden \\ Comprehensive Cancer Center, \\ University of lowa, lowa City, IA, \\ USA; ${ }^{3}$ Department of Pathology, \\ College of Medicine, University of \\ lowa, lowa City, IA, USA; ${ }^{4}$ Cancer \\ Biology Graduate Program, College \\ of Medicine, University of lowa, \\ lowa City, IA, USA; ${ }^{5}$ Medical Scientist \\ Training Program, College of Medicine, \\ University of lowa, lowa City, IA, USA
}

Correspondence: Praveen Vikas Holden Comprehensive Cancer Center, University of lowa 5983 JPP, 200 Hawkins Drive, lowa City, IA 52242, USA

Tel +I 3193562757

Email praveen-vikas@uiowa.edu

\begin{abstract}
Triple-negative breast cancer (TNBC) is a heterogeneous disease with poorer outcomes compared to other breast cancer subtypes. Contributing to the worse prognosis in TNBC is the higher rates of relapse and rapid progression after relapse. Advances in targeted therapeutics and conventional chemotherapy for TNBC have been stymied due to the lack of specific targets. Moreover, the responses to chemotherapy in TNBC lack durability, partially accounting for the higher rates of relapse. Immunotherapy, notably immune-checkpoint blockade, has shown to improve survival and maintain robust antitumor responses in both hematologic and solid malignancies. Unlike lung cancer, melanoma, and bladder cancer, most breast cancers are not inherently immunogenic and typically have low $\mathrm{T}$ cell infiltration. However, among breast cancer subtypes, TNBC is characterized by greater tumor immune infiltrate and higher degree of stromal and intratumoral tumor-infiltrating lymphocytes (TILs), a predictive marker for responses to immunotherapy. Moreover, in TNBC, the high number of stromal TILs is predictive of more favorable survival outcomes and response to chemotherapy. Immunotherapy is being extensively explored in TNBC and clinical trials are showing some promising results. This article focuses on the rationale for immunotherapy in TNBC, to explore and discuss preclinical data, results from early clinical trials, and to summarize some ongoing trials. We will also discuss the potential application of immunotherapy in TNBC from a clinician's perspective.
\end{abstract}

Keywords: triple-negative breast cancer, immunotherapy, PD-1/PDL-1 antibody, CTLA-4 antibody, checkpoint inhibitors, cancer vaccines

\section{Introduction}

Triple-negative breast cancer (TNBC) is a subtype of breast cancer characterized by absence of estrogen receptor (ER), progesterone receptor, and human epidermal growth factor receptor 2 (HER2) and represents about $15 \%-20 \%$ of all breast cancer. ${ }^{1}$ TNBC patients often present with visceral involvement and typically show rapid progression and thus providing limited window of treatment opportunity. Tremendous advances have occurred in other subtypes of breast cancer such as HER2+ tumors with the development of targeted agents against the HER2 receptor. Monoclonal antibodies, such as trastuzumab and pertuzumab and drug-antibody conjugates, like T-DM1, have changed the management of HER2+ breast cancer. ${ }^{2}$ ER + breast cancers have agents that block estrogen signaling through either aromatase inhibition or selective ER modulation. Coupling the anti-estrogen therapy with newly approved cyclin-dependent kinase inhibitors, like palbociclib, ribociclib, and abemaciclib, has shown improvements in progression-free survival. ${ }^{3,4}$ In an effort to discover actionable targets and drug 
development, TNBC has been further stratified into molecular subtypes. ${ }^{5}$ Gene expression profiling has revealed six distinct molecular subtypes, including two basal-like, an immunomodulatory, a mesenchymal, a mesenchymal stem-like, and a luminal androgen receptor. ${ }^{5}$ These molecular subtypes could be utilized in identifying personalized treatment strategies as they appear to have both predictive and prognostic value. ${ }^{5}$

Tumor-infiltrating lymphocytes (TILs), present both intratumorally and in adjacent stromal tissues, have predictive and prognostic roles in breast cancer. Within breast cancer, increased TILs at diagnosis have been significantly associated with pathologic complete responses with neoadjuvant chemotherapy. ${ }^{6,7}$ Interestingly, the improved overall survival in the context of neoadjuvant therapy was only seen in TNBC and HER2+ breast cancer and this improved prognosis was inversely associated with disease stage. ${ }^{6,7}$ This subtypespecific disparity in overall survival may be due to elevated TILs in TNBC compared to other breast cancer subtypes. ${ }^{8}$ In the context of HER2+ breast cancer, increased levels of TILs have been associated with improved response to the anti-HER2 antibody, trastuzumab. ${ }^{9}$ In subsequent work, a high degree of TILs found in majority of TNBC have been correlated with higher likelihood of benefit from adjuvant and neoadjuvant chemotherapy, with lower risk of disease relapse. ${ }^{9-13}$ This association of TILs in TNBC with therapeutic response and both overall and disease-free survival underscores the prognostic value of antitumor immunity in treating patients with TNBC. The presence of TILs also serves as a predictive marker of immunotherapy response, making the examination of immunotherapy in TNBC particularly intriguing. ${ }^{14}$ However, important consideration should be made for TNBC subtypes, with IM and basal-like subtypes possessing elevated infiltration of immune cells, antigen-presenting cells, and activation of immune pathways. ${ }^{15}$ Additionally, basal-like TNBC subtypes are known to have high frequency of $B R C A 1$ and $B R C A 2$ mutations and hence unstable genetics, another predictive marker for immunotherapy response. ${ }^{5,14}$

The immune system not only plays a role in tumor initiation and progression, but also participates in recognition and destruction of cancer cells. The antitumor immune response dampens development/progression through tumor-directed immune responses involving cytolytic T lymphocytes. ${ }^{16,17}$ For progression to occur, tumors must evade the cytotoxic antitumor response through a diverse array of mechanisms. The evasion of immune activation is recognized as a hallmark of cancer and can involve chronic activation of humoral immunity, infiltration by $\mathrm{Th} 2 \mathrm{~T}$ cells, protumor-polarized innate inflammatory cells, downregulation of tumor-specific anti- gens, expression of negative immune checkpoints by tumors, and the absence of major histocompatibility complexes (MHC) on the surface of tumors cells. ${ }^{14,16,17}$ Ultimately, these mechanisms act in concert to moderate antitumor response and promote tumor development and disease progression. ${ }^{18}$

Manipulation of the immune system represents an attractive strategy for TNBC, a breast cancer subtype that has not seen substantial advances in clinical management. Immunecheckpoint inhibitors have yielded promising results in both advanced and early-stage disease of TNBC patients and are expected to substantially improve the overall prognosis of TNBC. A focus of the field in translating immunotherapies from immunogenic tumors, like melanoma or renal cancers, to the majority of solid tumors is the identification of patients who would benefit most from immunotherapy and identification of agents to prime the tumor microenvironment. ${ }^{14}$ Of particular interest in the clinical management of TNBC would be the use of radiation or chemotherapy to augment responses to immunotherapy. Radiation increases mutational load of tumors, optimizes antigen presentation, and may act to decrease immune suppressors in the tumor microenvironment, priming the tumor for immunotherapy. ${ }^{19-23}$ In addition to radiation, recent investigation into the use of platinumbased chemotherapy before the induction of immunotherapy in lung cancer has shown superior response and progressionfree survival compared to the standard of care. ${ }^{24,25}$ In this review, we will comprehensively summarize early and ongoing clinical trials of immunotherapy in TNBC. Our goal is to offer a clinical prospective on the potential promise and perils of translating immunotherapies into TNBC and likely some other nonimmunogenic solid cancers.

\section{Immunotherapy}

Immunotherapy works through stimulation of the immune system by active immunization with cancer vaccines, or passive immunization through tumor-specific antibodies and immune modulators, like immune-checkpoint inhibitors.

\section{Immune-checkpoint inhibitors}

Immune checkpoints are a diverse set of regulatory points for the adaptive immune system, with roles in self-tolerance and antitumor immunity (Figure 1A). Physiologically, these checkpoints function in either the negative or positive regulation of the immune response, coordinating the magnitude and type of response. ${ }^{26}$ The majority of published clinical trials using immune-checkpoint blockade have focused on antibodies that target cytotoxic $\mathrm{T}$ lymphocyte-associated antigen 4 (CTLA-4) and the programmed cell death protein 

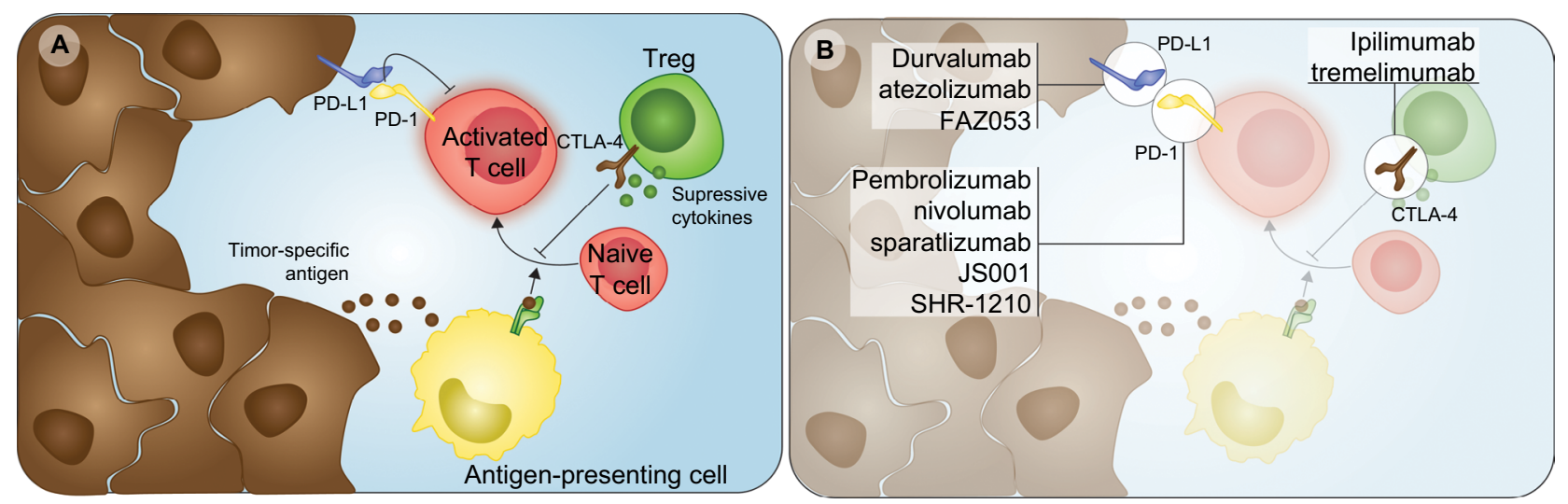

Figure I General mechanisms and agents targeting immune checkpoints in TNBC.

Notes: (A) Major immune cell players and interactions in the tumor microenvironment regulated by immune checkpoints. (B) Current therapies targeting PD-LI, PD-I, and CTLA-4 immune checkpoints under investigation in triple-negative breast cancer.

Abbreviations: CTLA-4, cytotoxic T lymphocyte-associated antigen 4; PD-I, programmed cell death protein I; PD-LI, programmed death ligand I; TNBC, triple-negative breast cancer; Treg, regulatory $T$ cell.

1 pathway (PD-1/PD-L1). As negative regulators of immune activation, the presence of CTLA-4 and PD-1 in the tumor microenvironment prevents a comprehensive antitumor immune response. ${ }^{26}$ Monoclonal antibodies targeting PD-1/ PD-L1 or CTLA-4 are thought to function by removing the inhibition of the antitumor immune response. ${ }^{26} \mathrm{~A}$ visual summary of agents targeting these two immune checkpoints that are currently being investigated in TNBC is available in Figure 1B.

PD-1 is expressed on the surface of lymphocytes and antigen-presenting cells. In the context of antitumor immunity, the binding of PD-1 on T cells with the ligands, PD-L1 or PD-L2, functions to suppress signals downstream of T-cell-receptor activation. ${ }^{27,28}$ With a lower side effect profile compared to anti-CTLA-4 and a wider range of approvals by the Food and Drug Administration (FDA), agents targeting PD-1 or PD-L1 have become a focus of moving immunecheckpoint blockade into many tumors. Several agents, such as pembrolizumab (anti-PD-1), nivolumab (anti-PD-1), atezolizumab (anti-PD-L1), avelumab (anti-PD-L1), and durvalumab (anti-PD-L1), have shown to be effective in various malignancies and are approved for treatment in melanoma, non-small-cell lung cancer, Hodgkin's lymphoma, bladder cancer, gastroesophageal cancer, primary mediastinal large B-cell lymphoma, cervical cancer, renal cancer, and head and neck cancer. ${ }^{29}$ Recent unprecedented approval of pembrolizumab in metastatic cancers with microsatellite instability or deficiency in DNA mismatch repair irrespective of the tissue of origin emphasizes the importance of mutational burden in response to immune-checkpoint blockade. ${ }^{30,31}$

PD-L1 expression has been found in 20\%-50\% of all breast cancer subtypes and its expression has been associated with higher histologic grades, larger tumors, and absence of hormone receptors. ${ }^{32}$ Higher PD-L1 expression has been observed in TNBCs as compared to non-TNBCs. ${ }^{33-35}$ This increased expression may be a result of genomic amplification of 9p24.1, containing CD274 (PD-L1) and PDCD1LG2 (PD-L2) in a subset of TNBC, but not ER+ tumors. ${ }^{36}$ Across a number of malignancies, the expression of PD-L1 has been associated with increased TILs and response to anti-PD-L1 immune-checkpoint blockade. ${ }^{37-44}$ In the context of the latter, PD-L1 staining in melanoma and lung cancer has been used to identify patients that would likely benefit from anti-PD-1/ PD-L1 therapies. ${ }^{45}$ Interestingly, the presence of PD-1+ lymphocytes and PD-L1 expression in breast cancer has been linked to poor prognosis in several reports. ${ }^{33,34}$ However, the immunohistochemical (IHC) staining of PD-1/PD-L1 was also tied to increased tumor stage, nodal involvement, triplenegative status, and Ki-67 proliferative marker staining, all independent poor prognostic indicators. ${ }^{33,34}$ More recently, the expression of PD-L1 in breast cancer, and specifically in basal-like TNBC, has been associated with longer overall and disease-free survival. ${ }^{46,47}$ The use of PD-L1 staining to segregate TNBC patients may be useful with increasing singleagent response rates from $5 \%-8.8 \%{ }^{48,49}$ to $18.5 \%-26 \% 0^{50-52}$ with the use of PD-L1 IHC staining as inclusion criteria.

\section{Pembrolizumab}

Receiving the initial FDA approval for unresectable or metastatic melanoma in 2014, pembrolizumab is one of the most studied of the immune-checkpoint therapies. Highlighting this fact was the presentation of clinical trial results in 25 tumor types at the 2018 annual meeting of the American Society of Clinical Oncology (ASCO). In 2016, 
the KEYNOTE-012 trial, Phase Ib study reported efficacy with acceptable safety profile when pembrolizumab was given $10 \mathrm{mg} / \mathrm{kg}$ every 2 weeks to patients with heavily pretreated, advanced TNBC. ${ }^{50}$ Among the 27 patients who were evaluated for antitumor activity, the overall response rate was $18.5 \%$, with median response time of 17.9 weeks. ${ }^{50} \mathrm{~A}$ singleagent Phase II study examining a $200 \mathrm{mg}$ dose given once every 3 weeks is ongoing. The KEYNOTE-086 trial is currently investigating the use of pembrolizumab in metastatic TNBC (NCT02447003) at $200 \mathrm{mg}$ every 3 weeks. In the oral session at the 2017 ASCO meeting, cohort A and B were presented. ${ }^{48,52}$ Cohort A consisted of TNBC patients that progressed on at least one systemic therapy. In this 170-patient cohort, $8(4.7 \%)$ responded and $13(7.6 \%)$ achieved disease control, to include stable disease, partial response, and complete response, for 24 weeks or longer. ${ }^{47}$ Additionally, one patient $(0.6 \%)$ had a complete response to pembrolizumab monotherapy, and $27 \%$ had an initial reduction in target lesion. ${ }^{47}$ Cohort B of the KEYNOTE-086 trial consisted of metastatic TNBC with PD-L1+ tumors, as defined by an IHC-based composite score, with no prior systemic therapy. In this cohort, objective responses were seen in $23 \%$ of 52 patients. ${ }^{52}$ The increased response in cohort $\mathrm{B}$ may be a result of the use of pembrolizumab as a first-line therapy and the use of PD-L1+ tumors as an inclusion criterion, with only $58 \%$ of the enrolled patients possessing a combined positive PD-L1 composite score of greater than $1 .^{52}$

Similar to recent success of combinatorial platinum and anti-PD-1 therapy in lung cancer, ${ }^{24,25}$ the use of DNA damaging agents may improve response by inducing cancer cell death and releasing tumor-specific antigens. In locally advanced breast cancer, the addition of pembrolizumab to standard neoadjuvant chemotherapy (paclitaxel followed by doxorubicin and cyclophosphamide) increased the rate of pathologic complete response approximately threefold in the I-SPY 2 trial. ${ }^{53}$ The addition of pembrolizumab to chemotherapy had a complete pathologic response of $60 \%$ vs $20 \%$ with chemotherapy-alone arm. ${ }^{53}$ Initial data on a small cohort of patients in the KEYNOTE-173 presented at ASCO 2017 suggested improved objective response rate of $100 \%$ with combination of pembrolizumab and carboplatin vs $80 \%$ in another experimental group (nab-paclitaxel + pembrolizumab).$^{54}$ In spontaneous tumor models of BRCA1-mutated TNBC, the use of cisplatin inhibited tumor growth and functioned additively with anti-PD-L1 and anti-CTLA-4 combinatorial therapy. ${ }^{55} \mathrm{~A}$ similar strategy of the use of polyADP ribose polymerase (PARP) inhibitors, selective singlestrand DNA inhibitors, in $B R C A$-mutated TNBC and ovarian cancer found a $45 \%$ objective response rate in combination with pembrolizumab, ${ }^{56}$ compared to the single-agent Phase I trial of the same PARP inhibitor (niraparib) response rate of $16.7 \%$, suggesting the combination of the two may function synergistically. ${ }^{57}$

\section{Anti-PD-LI therapies}

Initial phase I findings of the anti-PD-L1 monoclonal antibody (MPDL3280A, atezolizumab) in metastatic TNBC were also recently reported. Of the nine patients evaluable for efficacy, the overall response rate was 33\% (one complete and two partial responders) among advance TNBC patients, $90 \%$ of whom were previously treated with more than two prior regimens and one-third of whom enrolled had visceral metastases. ${ }^{58}$ Similarly, a larger-scale Phase Ib study involving 168 patients, with 57 TNBC patients, who previously received taxane and anthracycline therapies, was used to evaluate the anti-PD-L1 agent (avelumab). ${ }^{49}$ Across the entire cohort, the objective response rate was $5.4 \%$ with an additional 40 patients developing stable disease. ${ }^{49}$ Importantly, TNBC patients with PD-L1 expression on immune cells had the clinical response as high as $44.4 \%$ vs $2.6 \%$ in those TNBC patients without the PD-L1-positive immune cells. ${ }^{49}$ Similar response rate of $48.4 \%$ was seen using durvalumab in conjunction with neoadjuvant GeparNeuvo (NCT02685059). ${ }^{59}$ The variable expression patterns of PD-L1 in a tumor led to investigations into antibody-drug conjugates (ADC) of antiPD-L1 with monomethyl auristatin, an antimitotic drug. ${ }^{60}$ Using an anti-PD-L1 antibody with enhanced activity for glycosylated PD-L1, Li et al demonstrated that the use of the ADC enhances efficacy of immunotherapy and increases bystander-killing of adjacent tumor cells. ${ }^{60}$

\section{Combination of checkpoint blockade}

CTLA-4 functions earlier in the $\mathrm{T}$ cell activation process and is a major suppressive mechanism of the regulatory $\mathrm{T}$ cell (Treg). ${ }^{26,61}$ Recent research has suggested that depletion of Tregs by anti-CTLA-4 therapy is one of the major reasons leading to therapeutic responses. ${ }^{62,63}$ The nonredundant function of PD-1 and CTLA-4 in eliciting and maintaining immune cell activation led to combinatory therapy blocking the two immune checkpoints. In both melanoma and lung cancer, the use of nivolumab and ipilimumab (anti-CTLA-4) antibody has demonstrated better responses compared to nivolumab or chemotherapy alone. ${ }^{64,65}$ Interestingly, the efficacy of such combination relies on cancer types. In lung cancer the combination of nivolumab and ipilimumab did not perform any better than nivolumab alone in progression- 
free and overall survival regardless of PD-L1 staining. ${ }^{65}$ This has not slowed down the exploration of combinatorial antiCTLA-4/PD-1 trials, with an ongoing Phase I/II open-label trial of nivolumab plus ipilimumab vs nivolumab alone for patients with metastatic solid tumors, which include those with TNBC (NCT01928394). Additionally, a single-arm Phase II study of durvalumab in combination with the antiCTLA-4, tremelimumab, is recruiting metastatic HER2breast cancer patients (NCT02536794).

The combination of immune-checkpoint blockade with other drugs or targeting other immune checkpoints is moving forward in breast cancer (Figure 2). LAG-3 is another negative checkpoint that binds to MHC II receptors and modulates antigen-presenting cells; IMP321 (LAG-3Ig), a competitive inhibitor for LAG-3/MHC II receptor binding, when combined with paclitaxel had objective response rate of $50 \%$ as a first-line therapy. ${ }^{66}$ Targeting LAG-3 is the basis of several clinical trials in breast cancer, being used as an adjuvant to paclitaxel (NCT02614833) and in conjunction with anti-PD-1 therapy in TNBC (NCT02460224 and NCT03250832). Preliminary results of anti-PD-1 with anti-LAG-3 therapy found an objective response of $40 \%$ in TNBC ${ }^{67}$ In contrast, OX-40 is a positive immune-checkpoint functioning in maintaining $\mathrm{T}$ cell proliferation after receptor activation and suppressing Treg function. ${ }^{68,69} \mathrm{~A}$ Phase I/II trial into the combination of agonistic monoclonal antibody combined with either
anti-PD-1 or anti-CTLA-4 therapies is recruiting advanced cancers including TNBC (NCT03241173). With a similar rationale, NKTR-214, a CD122 agonist, is being evaluated in TNBC with initial result of a $33 \%$ response rate when paired with anti-PD-1 therapy. ${ }^{70}$ In contrast, the targeting of tumor-associated macrophages with anti-CSF1 and anti-PD-1 therapy had an objective response rate of $3.3 \%$ in a Phase $\mathrm{Ib}$ trial, underscoring the importance of selecting the right combinatorial agents for immune-checkpoint blockade. ${ }^{71}$ Comprehensive summaries of the diverse range of related Phase I and II trials are listed as in the neoadjuvant (Table 1) and adjuvant (Table 2) settings. Additionally, small-molecule inhibitors against growth factors, PARP inhibitors, other immune checkpoints, and/or radiotherapy are currently recruiting advanced-stage TNBC patients in combination with anti-PD-1/PD-L1 or anti-CTLA-4 antibodies (Table 3).

\section{Combining radiotherapy/cryotherapy with immune- checkpoint blockade}

Radiotherapy has potential to increase tumor mutation burden and increase tumor immunogenicity. ${ }^{21-23}$ The combination of radiotherapy with immune-checkpoint blockade has been studied in several tumor types like prostate cancer, lung cancer, sarcoma, and melanoma. ${ }^{21,23,72,73}$ The combination of local radiation to primary breast tumor with CTLA-4 blockade and PD-1/PD-L1 blockade has shown synergistic activity in

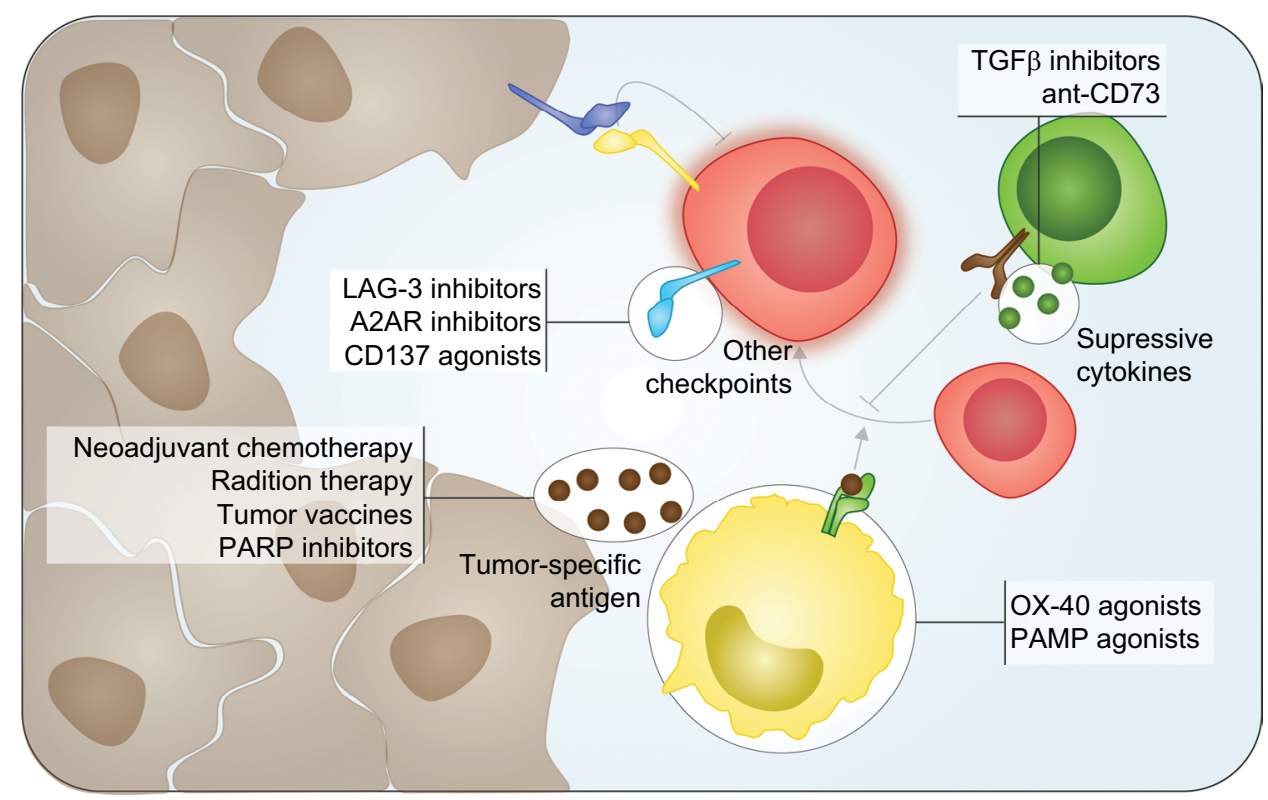

Figure 2 Emerging immunotherapy targets and combinatorial agents in TNBC.

Notes: Current trials focus on modulating the tumor microenvironment by increasing mutational burden in tumors, stimulating antigen-presenting cells, decreasing suppressive functions of Tregs, and targeting other negative immune checkpoints on effector $T$ cells.

Abbreviations: PAMP, pathogen-associated molecular pattern; PARP, poly-ADP ribose polymerase; TGF $\beta$, transforming growth factor beta; TNBC, triple-negative breast cancer; Treg, regulatory $\mathrm{T}$ cell. 
Table I Clinical trials using immunotherapy with neoadjuvant chemotherapy

\begin{tabular}{|l|l|l|l|l|l|}
\hline Clinical trial & Agent & Target & Combinatorial agent & Phase & $\begin{array}{l}\text { Recruitment } \\
\text { status }\end{array}$ \\
\hline NCT02983045 & Nivolumab & PD-I & NKTR-2I4 (CDI22), ipilimumab (CTLA-4) & I/II & Recruiting \\
NCT02489448 & Durvalumab & PD-LI & nab-Paclitaxel, doxorubicin, cyclophosphamide & I/II & Recruiting \\
NCT02685059 & Durvalumab & PD-LI & nab-Paclitaxel, epirubicin, cyclophosphamide & II & Active, not recruiting \\
NCT032898I9 & Pembrolizumab & PD-I & nab-Paclitaxel, epirubicin, cyclophosphamide & II & Recruiting \\
NCT03036488 & Pembrolizumab & PD-I & Carboplatin, paclitaxel, doxorubicin, & III & Recruiting \\
& & & epirubicin, cyclophosphamide & Recruiting & III \\
NCT0328I954 & Atezolizumab & PD-LI & Paclitaxel, doxorubicin, cyclophosphamide & Recling \\
\hline
\end{tabular}

Abbreviations: CTLA-4, cytotoxic T lymphocyte-associated antigen 4; PD-I, programmed cell death protein I; PD-LI, programmed death ligand I.

Table 2 Clinical trials using adjuvant immunotherapy

\begin{tabular}{|l|l|l|l|l|l|}
\hline Clinical trial & Agent & Target & Combinatorial agent & Phase & Recruitment status \\
\hline NCT03487666 & Nivolumab & PD-I & Capecitabine & II & Recruiting \\
NCT02926I96 & Avelumab & PD-LI & None & III & Recruiting \\
NCT02954874 & Pembrolizumab & PD-I & None & III & Recruiting \\
\hline
\end{tabular}

Abbreviations: PD-I, programmed cell death protein I; PD-LI, programmed death ligand I.

preclinical murine models. ${ }^{19,20}$ Current investigations into the use of combinatorial radiotherapy and immune-checkpoint blockade include a Phase II trial of pembrolizumab plus radiotherapy in metastatic TNBC (NCT02730130). This trial focuses on the benefit or harm of radiation concurrently with pembrolizumab. Similarly, a Phase II trial of nivolumab after radiotherapy induction or chemotherapy (low-dose doxorubicin, cyclophosphamide, or cisplatin) in patients with metastatic TNBC (NCT02499367) is being sponsored by the Netherlands Cancer Institute. Recent Phase II findings by the group have called into question of pairing these therapies with immune-checkpoint blockade, with an initial report that irradiation and/or cyclophosphamide halved the response rate compared to nivolumab alone. ${ }^{74}$ Other combinatory pilot studies have started, including the combination of stereotactic radiosurgery for oligometastatic breast cancer with pembrolizumab (NCT02303366) and the combination of hypofractionated radiotherapy with pembrolizumab in patients with advanced cancers, including breast cancer (NCT02303990).

\section{Cancer vaccine}

Cancer vaccines constitute another novel strategy in cancer immunotherapy. Therapeutic cancer vaccines have potential to elicit immune response against tumor-specific and tumorassociated antigens. Cancer vaccines include monovalent vaccines that provide a single tumor-associated antigen (TAA) target for the immune system and polyvalent peptide vaccines that provide multiple TAA targets. Cellular vaccines are mod- ified tumor cells or antigen-presenting cells. A current list of clinical trials in TNBC using tumor vaccines or oncolytic viruses with checkpoint blockade is summarized in Table 4. The use of polyvalent and antigen-specific vaccines offers the potential of truly precision therapy by using the mutations or drivers of a tumor to elicit an immune response. In high-risk breast cancers that include TNBC, cancer vaccines could play a role in preventing relapse; however, to date, multiple cancer vaccine trials have failed to show improvement in clinical outcome. Notably in a phase III trial of metastatic breast cancer, the targeting of sialyl-Tn, a carbohydrate antigen found on glycoproteins, demonstrated no improvements in progression-free interval or overall survival. ${ }^{75}$ In a follow-up analysis of results from the same trial, the authors did identify improvement in progression-free interval, overall survival, and antibody titer response in a subset of the patients receiving concomitant endocrine therapy. ${ }^{76}$

The use of antigen-specific vaccine strategies provides a selection pressure for tumors to evolve mechanisms to evade the narrow epitope targeting. ${ }^{77}$ One way of possibly overcoming this evasion is the use of personalized vaccines based on neoantigen fitness, or the likelihood of the antigen to be presented by the MHC and activate T cells. Although a number of barriers exist to prevent personalized vaccine usage for broad spectrum of patients in real time, the selection of multiple antigens based on the predicted ability to activate the antitumor immune system has shown promise in melanoma. ${ }^{78-82}$ When paired with anti-PD-1 therapy, the vaccines work synergistically with immune-checkpoint blockade..$^{79,81}$ 
Table 3 Immunotherapy in combination with various agents under investigation in advanced triple-negative disease

\begin{tabular}{|c|c|c|c|c|c|}
\hline Clinical trial & Agent & Target & Combinatorial agent & Phase & $\begin{array}{l}\text { Recruitment } \\
\text { status }\end{array}$ \\
\hline NCT02655822 & CPI-444 & A2AR & Atezolizumab (PD-LI) & I & Recruiting \\
\hline NCT0345445 I & CPI-006 & CD73 & Pembrolizumab (PD-I), CPI-444 (A2AR) & 1 & Recruiting \\
\hline NCT0325I3I3 & JSOOI & PD-I & Gemcitabine and cisplatin & I & Recruiting \\
\hline NCT030I 2230 & Pembrolizumab & PD-I & Ruxolitinib (JAK) & I & Recruiting \\
\hline NCT02890069 & Spartalizumab & PD-I & $\begin{array}{l}\text { Everolimus (mTOR), panobinostat (HDAC), } \\
\text { LCLI6I (apoptosis), QBM076 (CXCR2) }\end{array}$ & I & Recruiting \\
\hline NCT03250832 & TSR-033 & LAG-3 & Anti-PD-I antibodies & 1 & Recruiting \\
\hline NCT02646748 & Pembrolizumab & PD-I & Itacitinib (JAK), INCB050465 (PI3K) & I & Recruiting \\
\hline NCT02947I65 & NIS793 & TGF $\beta$ & Spartalizumab (PD-I) & 1 & Recruiting \\
\hline NCT03549000 & NZV930 & CD73 & Spartalizumab (PD-I), NIRI78 (A2AR) & I & Not yet recruiting \\
\hline NCT02838823 & JSOOI & PD-I & & 1 & Active, not recruiting \\
\hline NCT02622074 & Pembrolizumab & PD-I & $\begin{array}{l}\text { nab-Paclitaxel, doxorubicin, } \\
\text { cyclophosphamide, carboplatin }\end{array}$ & 1 & Active, not recruiting \\
\hline NCT03292172 & Atezolizumab & PD-LI & RO68708I0 (BET) & 1 & Recruiting \\
\hline NCT02936I02 & FAZ053 & PD-LI & Spartalizumab (PD-I) & I & Recruiting \\
\hline NCT03579472 & M7824 & PD-LI/TGF $\beta$ & Eribulin & I & Recruiting \\
\hline NCT0280744 & MCSIIO & CSF-I & Spartalizumab (PD-I) & I & Recruiting \\
\hline NCT02460224 & LAG525 & LAG-3 & Spartalizumab (PD-I) & $\mathrm{I} / \mathrm{II}$ & Recruiting \\
\hline NCT0324II73 & INCAGN0I949 & OX-40 & $\begin{array}{l}\text { Nivolumab (anti-PD-I) and/or ipilimumab } \\
\text { (anti-CTLA-4) }\end{array}$ & $1 / I I$ & Recruiting \\
\hline NCT0359I 276 & Pembrolizumab & PD-I & Pegylated doxorubicin & $\mathrm{I} / \mathrm{II}$ & Not yet recruiting \\
\hline NCT02628I32 & Durvalumab & PD-LI & Paclitaxel & $1 / I I$ & Recruiting \\
\hline NCT02657889 & Pembrolizumab & PD-I & Niraparib (PARP) & $1 / I I$ & Active, not recruiting \\
\hline NCT03356860 & Durvalumab & PD-LI & Paclitaxel, epirubicin, cyclophosphamide & $\mathrm{I} / \mathrm{II}$ & Recruiting \\
\hline NCT025I3472 & Pembrolizumab & PD-I & Eribulin & $\mathrm{I} / \mathrm{II}$ & Recruiting \\
\hline NCT02484404 & Durvalumab & PD-LI & Olaparib (PARP) & $1 / I I$ & Recruiting \\
\hline NCT02708680 & Atezolizumab & PD-LI & Entinostat (HDAC) & $1 / I I$ & Recruiting \\
\hline NCT02734004 & Durvalumab & PD-LI & Olaparib (PARP) & $1 / I I$ & Recruiting \\
\hline NCT026I4833 & IMP32I & LAG-3 & Paclitaxel & II & Recruiting \\
\hline NCT03394287 & SHR-1210 & PD-I & Apatanib (VEGFR) & II & Recruiting \\
\hline NCT034I4684 & Nivolumab & PD-I & Carboplatin & II & Recruiting \\
\hline NCT02648477 & Pembrolizumab & PD-I & Doxorubicin & II & Recruiting \\
\hline NCT03004I83 & Pembrolizumab & PD-I & SBRT and ADV/HSV-tk & II & Recruiting \\
\hline NCT02536794 & Durvalumab & PD-LI & Tremelimumab (CTLA-4) & II & Recruiting \\
\hline NCT02752685 & Pembrolizumab & PD-I & nab-Paclitaxel & II & Recruiting \\
\hline NCT03095352 & Pembrolizumab & PD-I & Carboplatin & II & Recruiting \\
\hline NCT03। 84558 & Pembrolizumab & PD-I & Bemcentinib (AXL) & II & Recruiting \\
\hline NCT0297I76I & Pembrolizumab & PD-I & $\begin{array}{l}\text { Enobosarm (selective androgen receptor } \\
\text { modulator) }\end{array}$ & II & Recruiting \\
\hline NCT025548I2 & Avelumab & PD-LI & Utomilumab (CDI37) & II & Recruiting \\
\hline NCT02849496 & Atezolizumab & PD-LI & Olaparib (PARP) & II & Recruiting \\
\hline NCT034830I 2 & Atezolizumab & PD-LI & Stereotactic radiosurgery & II & Recruiting \\
\hline NCT03I 64993 & Atezolizumab & PD-LI & Pegylated doxorubicin & II & Recruiting \\
\hline NCT0I898II7 & Atezolizumab & PD-LI & $\begin{array}{l}\text { Carboplatin and cyclophosphamide or } \\
\text { paclitaxel }\end{array}$ & II & Recruiting \\
\hline NCT02883062 & Atezolizumab & PD-LI & Carboplatin & II & Recruiting \\
\hline NCT0298I303 & Pembrolizumab & PD-I & Imprime PGG (PAMP) & II & Recruiting \\
\hline NCT028I95I8 & Pembrolizumab & PD-I & nab-Paclitaxel, gemcitabine, carboplatin & III & Active, not recruiting \\
\hline NCT034987I6 & Atezolizumab & PD-LI & Paclitaxel, epirubicin, cyclophosphamide & III & Recruiting \\
\hline NCT03। 97935 & Atezolizumab & PD-LI & $\begin{array}{l}\text { nab-Paclitaxel, doxorubicin, } \\
\text { cyclophosphamide }\end{array}$ & III & Recruiting \\
\hline NCT0242589I & Atezolizumab & PD-LI & nab-Paclitaxel & III & Active, not recruiting \\
\hline NCT03 I 25902 & Atezolizumab & PD-LI & Paclitaxel & III & Recruiting \\
\hline
\end{tabular}

Abbreviations: CTLA-4, cytotoxic T lymphocyte-associated antigen 4; HDAC, histone deacetylase; mTOR, mammalian target of rapamycin; PAMP, pathogen-associated molecular pattern; PARP, poly-ADP ribose polymerase; PD-I, programmed cell death protein I; PD-LI, programmed death ligand I; TGF $\beta$, transforming growth factor beta; VEGFR, vascular endothelial growth factor receptor. 
Table 4 Cancer vaccine and immunotherapy clinical trials currently being conducted

\begin{tabular}{|l|l|l|l|l|}
\hline Clinical trial & Agent & Combinatorial agent & Phase & Recruitment status \\
\hline NCT03362060 & PVX-4I0 & Pembrolizumab (PD-I) & I & Recruiting \\
NCT02826434 & PVX-4I0 & Durvalumab (PD-LI) & I & Recruiting \\
NCT03256344 & T-VEC & Atezolizumab (PD-LI) & I & Recruiting \\
NCT03289962 & RO7I98457 & Atezolizumab (PD-LI) & I & Recruiting \\
NCT01986426 & LTX-3I5 & Pembrolizumab (PD-I), ipilimumab (CTLA-4) & I & Active, not recruiting \\
NCT02432963 & P53MVA & Pembrolizumab (PD-I) & I & Active, not recruiting \\
NCT02779855 & T-VEC & Paclitaxel & I/II & Recruiting \\
NCT03387085 & NANT & Aldoxorubicin, avelumab (PD-LI), bevacizumab (VEGF), capecitabine, & I/II & Recruiting \\
& & cisplatin, cyclophosphamide, fluorouracil, nab-paclitaxel & & Recruiting \\
NCT03328026 & SV-BR-I-GM & Pembrolizumab (PD-I), ipilimumab (CTLA-4) & I/II \\
\hline
\end{tabular}

Abbreviations: CTLA-4, cytotoxic T lymphocyte-associated antigen 4; PD-I, programmed cell death protein I; PD-LI, programmed death ligand I; VEGF, vascular endothelial growth factor.

Similarly, the use of polyvalent vaccines derived from multiple antigens of the tumor or cell line has shown positive results in colorectal cancer, ${ }^{83}$ prostate cancer, ${ }^{84}$ follicular lymphoma ${ }^{85}$ and lung cancer. ${ }^{86}$ The high rates of genomic instability within TNBC make the neoantigen fitness-based and polyvalent vaccination strategies intriguing.

Another avenue is the immunization of tumors with virus, such as the use of vaccinia virus modified to express MUC1 and interleukin 2. ${ }^{87}$ The viral-mediated expression of MUC1, a nonspecific tumor antigen, to elicit an antitumor immune response mirrors the sialyl-Tn vaccination strategy. ${ }^{75}$ Although partial tumor regression occurred in only 2 of the 31 patients with metastatic breast cancer, 14 patients developed stable disease.$^{87} \mathrm{~A}$ similar strategy of creating a tumorspecific antigen using a virus is being investigated in using a Modified Vaccinia Ankara virus to re-express wild-type p53 in combination with pembrolizumab. ${ }^{88}$ Additionally, several small pilot studies of the oncolytic herpes simplex virus, HF10, demonstrated significant histopathologic response and reduction in tumor size of $30 \%-100 \%{ }^{89,90}$ The use of HF 10 is currently being investigated in combination with chemotherapy for treatment of patients with unresectable pancreatic cancer (NCT03252808). Like these cancer-specific viral inoculations, newer vaccine approaches are on the horizon that can simultaneously vaccinate and use immune-checkpoint blockers to therapeutically block immune-suppressive pathway, which may increase the objective response rates.

\section{Conclusion}

TNBC remains the breast cancer subtype with the worse outcomes. Despite the progress made across many other cancers and other subtypes of breast cancer, effective treatment for advanced TNBC remains elusive. Immunotherapy with checkpoint inhibitors has shown promise, but response rates are very modest as single agent in advanced TNBCs. We have seen durable response in early-phase trials with anti-PD1 or anti-PD-L1, but response rates are up to $10 \%$ in unselected TNBC patients and improves only slightly to $20 \%-30 \%$ when patients are selected based on IHC-based PD-L1+ tumors. ${ }^{48-52}$

TILs appear to be higher in TNBC and multiple series of investigations have shown that higher TILs are associated with improved overall survival and higher response rate. ${ }^{9-13}$ The immunogenic microenvironment is associated with a better prognosis as a result of a higher likelihood of benefit from chemotherapy and possibly from immunotherapy, whereas cold immune microenvironment carries greater risk of relapse and lower benefit from chemotherapy and possibly immunotherapy. ${ }^{6,714}$ Identifying the subset of TNBC who will benefit most from available means of immune modulation should remain the priority while new targets and drugs are being developed. Biomarker-based patient identification, such as $B R C A$ mutation, mutations in homologous recombination, and PD-L1 IHC testing, may improve clinical responses, but effort needs to focus on improving the development and standardization of valuable biomarkers of response. ${ }^{49,55,56}$

Akin to the identification of biomarkers for immunotherapy response in TNBC, investigations are currently underway for combinatorial agents used as short-term induction treatment to increase immunogenicity in TNBC. Careful consideration of combination strategies, like the use of PARP inhibitors or specific chemotherapies, could be a highly successful strategy for immunotherapy in TNBC.

The development and early-phase trials into new immune checkpoints and tumor vaccine strategies for TNBC are compounding the excitement in the field. For a cancer that has not seen substantial advances in clinical management in recent decades, immunotherapy gives us a hope for durable 
clinical response for patients with TNBC. New agents and new combinations of immunotherapies may unlock the key to truly personalized cancer medicine. Belying the promise of immunotherapy in TNBC is a number of steps required to translate these therapies into meaningful outcomes and ultimately could turn out to be a hype. Specifically, efforts focused on understanding biology, biomarker selection, and strategies to enhance immunotherapy response are vital to the success of immunotherapy in TNBC and cancers in general.

\section{Abbreviations}

TNBC, triple-negative breast cancer; TILs, tumor-infiltrating lymphocytes; ER, estrogen receptor; PR, progesterone receptor; HER2, human epidermal growth factor receptor 2; MHC, major histocompatibility complexes; CTLA-4, cytotoxic $\mathrm{T}$ lymphocyte-associated antigen 4; PD-1, programmed cell death protein 1; IHC, immunohistochemical; ASCO, American Society of Clinical Oncology; PARP, poly-ADP ribose polymerase; ADC, antibody-drug conjugates; Treg, regulatory $\mathrm{T}$ cell; TAA, tumor-associated antigen.

\section{Acknowledgments}

The authors are supported by the National Cancer Institute of the National Institutes of Health under award numbers R01s CA200673 (WZ), CA203834 (WZ), and F30 fellowship CA206255 (NB).

\section{Disclosure}

The authors report no conflicts of interest in this work.

\section{References}

1. Cancer Genome Atlas Network. Comprehensive molecular portraits of human breast tumours. Nature. 2012;490(7418):61-70.

2. Dawood S, Broglio K, Buzdar AU, Hortobagyi GN, Giordano SH. Prognosis of women with metastatic breast cancer by HER2 status and trastuzumab treatment: an institutional-based review. J Clin Oncol. 2010;28(1):92-98

3. Cristofanilli M, Turner NC, Bondarenko I, et al. Fulvestrant plus palbociclib versus fulvestrant plus placebo for treatment of hormonereceptor-positive, HER2-negative metastatic breast cancer that progressed on previous endocrine therapy (PALOMA-3): final analysis of the multicentre, double-blind, phase 3 randomised controlled trial. Lancet Oncol. 2016;17(4):425-439.

4. DiPippo AJ, Patel NK, Barnett CM. Cyclin-dependent kinase inhibitors for the treatment of breast cancer: past, present, and future. Pharmacotherapy. 2016;36(6):652-667.

5. Lehmann BD, Bauer JA, Chen X, et al. Identification of human triplenegative breast cancer subtypes and preclinical models for selection of targeted therapies. J Clin Invest. 2011;121(7):2750-2767.

6. Loi S. Tumor-infiltrating lymphocytes, breast cancer subtypes and therapeutic efficacy. Oncoimmunology. 2013;2(7):e24720.

7. Adams S, Gray RJ, Demaria S, et al. Prognostic value of tumor-infiltrating lymphocytes in triple-negative breast cancers from two phase III randomized adjuvant breast cancer trials: ECOG 2197 and ECOG 1199. J Clin Oncol. 2014;32(27):2959-2966.
8. García-Teijido P, Cabal ML, Fernández IP, Pérez YF. Tumor-infiltrating lymphocytes in triple negative breast cancer: the future of immune targeting. Clin Med Insights Oncol. 2016;10(Suppl 1):31-39.

9. Loi S, Michiels S, Salgado R, et al. Tumor infiltrating lymphocytes are prognostic in triple negative breast cancer and predictive for trastuzumab benefit in early breast cancer: results from the FinHER trial. Ann Oncol. 2014;25(8):1544-1550.

10. Adams S, Goldstein LJ, Sparano JA, Demaria S, Badve SS. Tumor infiltrating lymphocytes (TILs) improve prognosis in patients with triple negative breast cancer (TNBC). Oncoimmunology. 2015;4(9):e985930-985933.

11. Denkert C, von Minckwitz G, Darb-Esfahani S, et al. Abstract S1-09: evaluation of tumor-infiltrating lymphocytes (TILs) as predictive and prognostic biomarker in different subtypes of breast cancer treated with neoadjuvant therapy - a metaanalysis of 3771 patients. Cancer Res. 2017;77(4 Supplement):S1-09.

12. Loi S, Sirtaine N, Piette F, et al. Prognostic and predictive value of tumor-infiltrating lymphocytes in a phase III randomized adjuvant breast cancer trial in node-positive breast cancer comparing the addition of docetaxel to doxorubicin with doxorubicin-based chemotherapy: BIG 02-98. J Clin Oncol. 2013;31(7):860-867.

13. Dieci MV, Criscitiello C, Goubar A, et al. Prognostic value of tumorinfiltrating lymphocytes on residual disease after primary chemotherapy for triple-negative breast cancer: a retrospective multicenter study. Ann Oncol. 2014;25(3):611-618.

14. Borcherding N, Kolb R, Gullicksrud J, Vikas P, Zhu Y, Zhang W. Keeping tumors in check: a mechanistic review of clinical response and resistance to immune checkpoint blockade in cancer. $J$ Mol Biol. 2018;430(14):2014-2029.

15. Vinayak S, Gray RJ, Adams S. Association of increased tumor-infiltrating lymphocytes (TILs) with immunomodulatory (IM) triple-negative breast cancer (TNBC) subtype and response to neoadjuvant platinumbased therapy in PrECOG0105. J Clin Oncol. 2017;32(15_suppl): 1000 .

16. Hanahan D, Weinberg RA. Hallmarks of cancer: the next generation. Cell. 2011;144(5):646-674.

17. de Visser KE, Coussens LM. The inflammatory tumor microenvironment and its impact on cancer development. Contrib Microbiol. 2006; 13:118-137.

18. Hagerling C, Casbon AJ, Werb Z. Balancing the innate immune system in tumor development. Trends Cell Biol. 2015;25(4):214-220.

19. Sharabi AB, Nirschl CJ, Kochel CM, et al. Stereotactic radiation therapy augments antigen-specific pd-1-mediated antitumor immune responses via cross-presentation of tumor antigen. Cancer Immunol Res. 2015;3(4):345-355.

20. Demaria S, Kawashima N, Yang AM, et al. Immune-mediated inhibition of metastases after treatment with local radiation and CTLA-4 blockade in a mouse model of breast cancer. Clin Cancer Res. 2005;11(2 Pt 1):728-734.

21. Twyman-Saint Victor C, Rech AJ, Maity A, et al. Radiation and dual checkpoint blockade activate non-redundant immune mechanisms in cancer. Nature. 2015;520(7547):373-377.

22. Sharabi AB, Lim M, Deweese TL, Drake CG. Radiation and checkpoint blockade immunotherapy: radiosensitisation and potential mechanisms of synergy. Lancet Oncol. 2015;16(13):e498-e509.

23. Takahashi Y, Yasui T, Tamari K, et al. Radiation enhanced the local and distant anti-tumor efficacy in dual immune checkpoint blockade therapy in osteosarcoma. PLoS One. 2017;12(12):e0189697.

24. Langer CJ, Gadgeel SM, Borghaei H, et al. Carboplatin and pemetrexed with or without pembrolizumab for advanced, non-squamous nonsmall-cell lung cancer: a randomised, phase 2 cohort of the open-label KEYNOTE-021 study. Lancet Oncol. 2016;17(11):1497-1508.

25. Gandhi L, Rodríguez-Abreu D, Gadgeel S. Pembrolizumab plus chemotherapy in metastatic non-small-cell lung cancer. $N$ Engl J Med. 2018;378(22):2078-2092.

26. Pardoll DM. The blockade of immune checkpoints in cancer immunotherapy. Nat Rev Cancer. 2012;12(4):252-264. 
27. Chemnitz JM, Parry RV, Nichols KE, June CH, Riley JL. SHP-1 and SHP-2 associate with immunoreceptor tyrosine-based switch motif of programmed death 1 upon primary human $\mathrm{T}$ cell stimulation, but only receptor ligation prevents $\mathrm{T}$ cell activation. J Immunol. 2004;173(2):945-954.

28. Sheppard KA, Fitz LJ, Lee JM, et al. PD-1 inhibits T-cell receptor induced phosphorylation of the ZAP70/CD3zeta signalosome and downstream signaling to PKCtheta. FEBS Lett. 2004;574(1-3):37-41.

29. Hargadon KM, Johnson CE, Williams CJ. Immune checkpoint blockade therapy for cancer: an overview of FDA-approved immune checkpoint inhibitors. Int Immunopharmacol. 2018;62:29-39.

30. Le DT, Uram JN, Wang H, et al. PD-1 blockade in tumors with mismatch-repair deficiency. N Engl J Med. 2015;372(26):2509-2520.

31. Le DT, Durham JN, Smith KN, et al. Mismatch repair deficiency predicts response of solid tumors to PD-1 blockade. Science. 2017;357(6349): 409-413.

32. Ghebeh H, Mohammed S, Al-Omair A, et al. The B7-H1 (PD-L1) T lymphocyte-inhibitory molecule is expressed in breast cancer patients with infiltrating ductal carcinoma: correlation with important high-risk prognostic factors. Neoplasia. 2006;8(3):190-198.

33. Muenst S, Soysal SD, Gao F, Obermann EC, Oertli D, Gillanders WE. The presence of programmed death 1 (PD-1)-positive tumor-infiltrating lymphocytes is associated with poor prognosis in human breast cancer. Breast Cancer Res Treat. 2013;139(3):667-676.

34. Muenst S, Schaerli AR, Gao F, et al. Expression of programmed death ligand 1 (PD-L1) is associated with poor prognosis in human breast cancer. Breast Cancer Res Treat. 2014;146(1):15-24.

35. Mittendorf EA, Philips AV, Meric-Bernstam F, et al. PD-L1 expression in triple-negative breast cancer. Cancer Immunol Res. 2014;2(4):361-370.

36. Barrett MT, Anderson KS, Lenkiewicz E, et al. Genomic amplification of 9p24.1 targeting JAK2, PD-L1, and PD-L2 is enriched in high-risk triple negative breast cancer. Oncotarget. 2015;6(28):26483-26493.

37. Taube JM, Anders RA, Young GD, et al. Colocalization of inflammatory response with B7-h1 expression in human melanocytic lesions supports an adaptive resistance mechanism of immune escape. Sci Transl Med. 2012;4(127):127ra37.

38. Taube JM, Klein A, Brahmer JR, et al. Association of PD-1, PD-1 ligands, and other features of the tumor immune microenvironment with response to anti-PD-1 therapy. Clin Cancer Res. 2014;20(19):5064-5074.

39. Taube JM, Young GD, McMiller TL, et al. Differential expression of immune-regulatory genes associated with PD-L1 display in melanoma: Implications for PD-1 pathway blockade. Clin Cancer Res. 2015;21(17):3969-3976.

40. Tumeh PC, Harview CL, Yearley JH, et al. PD-1 blockade induces responses by inhibiting adaptive immune resistance. Nature. 2014;515(7528):568-571

41. Ansell SM, Lesokhin AM, Borrello I, et al. PD-1 blockade with nivolumab in relapsed or refractory Hodgkin's lymphoma. $N$ Engl $J$ Med. 2015;372(4):311-319.

42. Brahmer J, Reckamp KL, Baas P, et al. Nivolumab versus docetaxel in advanced squamous-cell non-small-cell lung cancer. $N$ Engl J Med. 2015;373(2):1627-1639.

43. Passiglia F, Bronte G, Bazan V, et al. PD-L1 expression as predictive biomarker in patients with NSCLC: a pooled analysis. Oncotarget. 2016;7(15):19738-19747.

44. Inoue $\mathrm{H}$, Park JH, Kiyotani $\mathrm{K}$, et al. Intratumoral expression levels of $P D-L 1, G Z M A$, and $H L A-A$ along with oligoclonal T cell expansion associate with response to nivolumab in metastatic melanoma. Oncoimmunology. 2016;5(9):e1204507.

45. Gandini S, Massi D, Mandalà M. PD-L1 expression in cancer patients receiving anti PD-1/PD-L1 antibodies: a systematic review and metaanalysis. Crit Rev Oncol Hematol. 2016;100:88-98.

46. Sun WY, Lee YK, Koo JS. Expression of PD-L1 in triple-negative breast cancer based on different immunohistochemical antibodies. $J$ Transl Med. 2016;14(1):173.

47. Sabatier R, Finetti P, Mamessier E, et al. Prognostic and predictive value of PDL1 expression in breast cancer. Oncotarget. 2015;6(7): $5449-5464$.
48. Adams S, Schmid P, Rugo HS, et al. Phase 2 study of pembrolizumab (pembro) monotherapy for previously treated metastatic triple-negative breast cancer (mTNBC): KEYNOTE-086 cohort A. J Clin Oncol. 2017;35(15_suppl):1008.

49. Dirix L, Takacs I, Nikolinakos P. Avelumab (MSB0010718C), an antiPD-L1 antibody, in patients with locally advanced or metastatic breast cancer: a phase Ib JAVELIN solid tumor trial. In: San Antonio Breast Cancer Symposium. 2014:S1-4.

50. Nanda R, Chow LQ, Dees EC, et al. Pembrolizumab in patients with advanced triple-negative breast cancer: Phase Ib KEYNOTE-012 study. J Clin Oncol. 2016;34(21):2460-2467.

51. Schmid P. Atezolizumab in metastatic TNBC (mTNBC): long-term clinical outcomes and biomarker analyses. In: AACR Proceedings. 2017:\#2986.

52. Adams S, Loi S, Toppmeyer D. Phase 2 study of pembrolizumab as first-line therapy for PD-L1-positive metastatic triple-negative breast cancer (mTNBC): preliminary data from KEYNOTE-086 cohort B. $J$ Clin Oncol. 2017;35(15_suppl):1088.

53. Nanda R, Liu MC, Yau C, et al. Pembrolizumab plus standard neoadjuvant therapy for high-risk breast cancer (BC): results from I-SPY 2. J Clin Oncol. 2017;35(15_suppl):506.

54. Schmid P, Park YH, Muñoz-Couselo E. Pembrolizumab (pembro) + chemotherapy (chemo) as neoadjuvant treatment for triple negative breast cancer (TNBC): preliminary results from KEYNOTE-173. PASCO. 2017;35(15_suppl):556.

55. Nolan E, Savas P, Policheni AN, et al. Combined immune checkpoint blockade as a therapeutic strategy for BRCA1-mutated breast cancer. Sci Transl Med. 2017;9(393):eaal4922.

56. Konstantinopoulos PA, Waggoner SE, Vidal GA, et al. TOPACIO/ Keynote-162 (NCT02657889): a phase 1/2 study of niraparib + pembrolizumab in patients (pts) with advanced triple-negative breast cancer or recurrent ovarian cancer (ROC) - results from ROC cohort. J Clin Oncol. 2018;36(15_suppl):106.

57. Sandhu SK, Schelman WR, Wilding G, et al. The poly (ADP-ribose) polymerase inhibitor niraparib (MK4827) in BRCA mutation carriers and patients with sporadic cancer: a phase 1 dose-escalation trial. Lancet Oncol. 2013;14(9):882-892.

58. Emens L, Braiteh F, Cassier P. Inhibition of PD-L1 by MPDL3280A leads to clinical activity in patients with metastatic triple-negative breast cancer. In: San Antonio Breast Cancer Symposium. 2014:PD1-PD16.

59. Loibl S, Untch M, Burchardi N, et al. Randomized phase II neoadjuvant study (GeparNuevo) to investigate the addition of durvalumab to a taxane-anthracycline containing chemotherapy in triple negative breast cancer (TNBC). J Clin Oncol. 2018;36(15_suppl):104.

60. Li CW, Lim SO, Chung EM, et al. Eradication of triple-negative breast cancer cells by targeting glycosylated PD-L1. Cancer Cell. 2018;33(2):e10:187-201.

61. Wing K, Onishi Y, Prieto-Martin P, et al. CTLA-4 control over Foxp3+ regulatory T cell function. Science. 2008;322(5899):271-275.

62. Selby MJ, Engelhardt JJ, Quigley M, et al. Anti-CTLA-4 antibodies of IgG2a isotype enhance antitumor activity through reduction of intratumoral regulatory T cells. Cancer Immunol Res. 2013;1(1):32-42.

63. Arce Vargas F, Furness AJS, Litchfield K, et al. Fc effector function contributes to the activity of human anti-CTLA-4 antibodies. Cancer Cell. 2018;33(4):649-663.

64. Larkin J, Chiarion-Sileni V, Gonzalez R, et al. Combined nivolumab and ipilimumab or monotherapy in untreated melanoma. $N$ Engl J Med. 2015;373(1):23-34.

65. Hellmann MD, Ciuleanu TE, Pluzanski A, et al. Nivolumab plus ipilimumab in lung cancer with a high tumor mutational burden. $N$ Engl $J$ Med. 2018;378(22):2093-2104.

66. Brignone C, Gutierrez M, Mefti F, et al. First-line chemoimmunotherapy in metastatic breast carcinoma: combination of paclitaxel and IMP321 (LAG-3Ig) enhances immune responses and antitumor activity. JTransl Med. 2010;8:71.

67. Hong DS, Schoffski P, Calvo A, et al. Phase I/II study of LAG525 \pm spartalizumab (PDR001) in patients (pts) with advanced malignancies. J Clin Oncol. 2018;36(15_suppl):3012 . 
68. Piconese S, Valzasina B, Colombo MP. OX40 triggering blocks suppression by regulatory $\mathrm{T}$ cells and facilitates tumor rejection. J Exp Med. 2008;205(4):825-839.

69. Linch SN, McNamara MJ, Redmond WL. OX40 agonists and combination immunotherapy: putting the pedal to the metal. Front Oncol. 2015;5:34

70. Diab A, Hurwitz ME, Cho DC, et al. NKTR-214 (CD122-biased agonist) plus nivolumab in patients with advanced solid tumors: preliminary phase 1/2 results of PIVOT. J Clin Oncol. 2018;363006(15_suppl):3006.

71. Calvo A, Joensuu H, Sebastian M, et al. Phase Ib/II study of lacnotuzumab (MCS110) combined with spartalizumab (PDR001) in patients (pts) with advanced tumors. JClin Oncol. 2018;363014(15_suppl):3014.

72. Kwon ED, Drake CG, Scher HI, et al; CA184-043 Investigators. Ipilimumab versus placebo after radiotherapy in patients with metastatic castration-resistant prostate cancer that had progressed after docetaxe chemotherapy (CA184-043): a multicentre, randomised, double-blind, phase 3 trial. Lancet Oncol. 2014;15(7):700-712.

73. Golden EB, Demaria S, Schiff PB, Chachoua A, Formenti SC. An abscopal response to radiation and ipilimumab in a patient with metastatic non-small cell lung cancer. Cancer Immunol Res. 2013;1(6):365-372.

74. Kok M, Voorwerk L, Horlings H, et al. Adaptive phase II randomized trial of nivolumab after induction treatment in triple negative breast cancer (TONIC trial): final response data stage I and first translational data. J Clin Oncol. 2018;36(15_suppl):1012.

75. Miles D, Roché H, Martin M, et al; Theratope ${ }^{\circledR}$ Study Group. Phase III multicenter clinical trial of the sialyl-TN (STn)-keyhole limpet hemocyanin $(\mathrm{KLH})$ vaccine for metastatic breast cancer. Oncologist. 2011;16(8):1092-1100.

76. Ibrahim NK, Murray JL, Zhou D, et al. Survival advantage in patients with metastatic breast cancer receiving endocrine therapy plus Sialyl Tn-KLH vaccine: post hoc analysis of a large randomized trial. J Cancer. 2013;4(7):577-584.

77. Melero I, Gaudernack G, Gerritsen W, et al. Therapeutic vaccines for cancer: an overview of clinical trials. Nat Rev Clin Oncol. 2014;11(9):509-524

78. Łuksza M, Riaz N, Makarov V, et al. A neoantigen fitness model predicts tumour response to checkpoint blockade immunotherapy. Nature. 2017;551(7681):517-520.

79. Ott PA, Hu Z, Keskin DB, et al. An immunogenic personal neoantigen vaccine for patients with melanoma. Nature. 2017;547(7662):217-221.
80. Linnemann C, van Buuren MM, Bies L, et al. High-throughput epitope discovery reveals frequent recognition of neo-antigens by CD4+ T cells in human melanoma. Nat Med. 2015;21(1):81-85.

81. Sahin U, Derhovanessian E, Miller M, et al. Personalized RNA mutanome vaccines mobilize poly-specific therapeutic immunity against cancer. Nature. 2017;547(7662):222-226.

82. Strønen E, Toebes M, Kelderman S, et al. Targeting of cancer neoantigens with donor-derived T cell receptor repertoires. Science. 2016;352(6291):1337-1341.

83. Vermorken JB, Claessen AM, van Tinteren H, et al. Active specific immunotherapy for stage II and stage III human colon cancer: a randomised trial. Lancet. 1999;353(9150):345-350.

84. Small E, Demkow T, Gerritsen WR, et al. A phase III trial of GVAX immunotherapy for prostate cancer in combination with docetaxel versus docetaxel plus prednisone in symptomatic, castration-resistant prostate cancer (CRPC). In: 2009 Genitourinary Cancers Symposium; 2009:07.

85. Schuster SJ, Neelapu SS, Gause BL, et al. Vaccination with patient-specific tumor-derived antigen in first remission improves disease-free survival in follicular lymphoma. J Clin Oncol. 2011;29(20):2787-2794.

86. Giaccone G, Bazhenova LA, Nemunaitis J. A phase III study of belagenpumatucel-L therapeutic tumor cell vaccine for non-small cell lung cancer (NSCLC). Eur J Cancer. 2013;49:S2-S3.

87. Scholl S, Squiban P, Bizouarne N, et al. Metastatic breast tumour regression following treatment by a gene-modified vaccinia virus expressing MUC1 and IL-2. J Biomed Biotechnol. 2003;2003(3):194-201

88. Chung VM, Kos F, Hardwick N, et al. A phase 1 study of p53MVA vaccine in combination with pembrolizumab. J Clin Oncol. 2018 ; 36(5_suppl):206.

89. Kimata H, Imai T, Kikumori T, et al. Pilot study of oncolytic viral therapy using mutant herpes simplex virus (HF10) against recurrent metastatic breast cancer. Ann Surg Oncol. 2006;13(8):1078-1084.

90. Sahin TT, Kasuya H, Nomura N, et al. Impact of novel oncolytic virus HF10 on cellular components of the tumor microenvironment in patients with recurrent breast cancer. Cancer Gene Ther. 2012;19(4):229-237.
Cancer Management and Research

\section{Publish your work in this journal}

Cancer Management and Research is an international, peer-reviewed open access journal focusing on cancer research and the optimal use of preventative and integrated treatment interventions to achieve improved outcomes, enhanced survival and quality of life for the cancer patient. The manuscript management system is completely online and includes
Dovepress

a very quick and fair peer-review system, which is all easy to use. Visit $\mathrm{http}: / / \mathrm{www}$. dovepress.com/testimonials.php to read real quotes from published authors. 